\title{
The formation of amyloid fibril-like hen egg-white lysozyme species induced by temperature and urea concentration-dependent denaturation
}

\author{
Steven S.-S. Wang ${ }^{\dagger}$, Ying-Tz Hung, Pu Wang and Josephine W. Wu \\ Department of Chemical Engineering, National Taiwan University, Taipei 10617, Taiwan \\ (Received 13 December $2006 \cdot$ accepted 4 March 2007)
}

\begin{abstract}
Recent evidence has suggested that the formation of partially unfolded denatured intermediate serves as a crucial prerequisite for protein fibril formation/aggregation. Despite extensive exploration on amyloid fibril formation, the detailed molecular mechanism has remained largely unknown. Here, we examined the effects of urea on the denaturation of hen egg-white lysozymes. Our results demonstrated that, in the solutions ( $\mathrm{pH} 7.4$ ) with $8 \mathrm{M}$ urea at all temperatures and with $4 \mathrm{M}$ urea at 45 or $55^{\circ} \mathrm{C}$, the amyloid fibril-like species were first produced and then vanished while such species were not observed under other conditions. In addition, SDS-PAGE results further indicated that larger aggregated species with less ordered structures were formed at the later stage of the urea-induced unfolding process owing to an unidentified conformational switch. We believe that the results in this work will aid in deciphering the molecular mechanism(s) of protein denaturation.
\end{abstract}

Key words: Lysozyme, Amyloid, Fibril, Aggregation, Urea, Partial Unfolding, Denaturation

\section{INTRODUCTION}

Protein folding, the process by which a linear sequence of amino acids forms a native protein exhibiting biological activity, is one of the most integral life processes, but its intricacy and complexity within living cells have only recently been appreciated. While the underlying mechanisms of folding are still rather elusive, investigators have recognized that protein folding reactions are complex mechanisms associated with numerous transient, partially folded or unfolded intermediates. Moreover, the correct folding of a protein probably involves temporary interactions with existing helper proteins or molecular chaperones, and is governed by evolutionary pressures that adjust the folding rates according to physiological requirements [1-4].

Incorrect folding or misfolding of proteins results in the formation of protein aggregates. Protein aggregates in general can be categorized into two types: amorphous aggregates, the ones with no long-range order, and amyloid fibrils, the ones with highly ordered structure. Amyloid fibrils, in vivo, underlie a vast array of diverse and debilitating human disorders, collectively known as the conformational diseases. These protein conformational diseases include the hemodialysis amyloidosis, type II diabetes, Parkinson's disease, Huntington's disease, and Alzheimer's disease [5-8]. These diseases individually have their unique pathological, clinical, and biochemical characteristics and their corresponding proteins have unrelated functions and exhibit little sequence or structural homology. Moreover, mounting evidence has suggested that the formation of amyloid fibrils is also possible to a variety of non-disease associated proteins [9-11]. The formation of fibrillar species in vitro as a generic property of proteins is gaining renewed attention [5,12-15]. The structure of amyloids is recognized to possess several specific

\footnotetext{
To whom correspondence should be addressed.

E-mail: sswang@ntu.edu.tw

Y. T. Hung and P. Wang both have an equal contribution to the manuscript.
}

tinctorial and physicochemical features in common: exhibition of $\beta$-sheet rich secondary structure, fibrillar morphology, birefringence upon staining with Congo red dye, insolubility in most solvents [58]. The striking, common feature of the proteins implicated in the conformational diseases is their ability to adopt at least two different stable conformations that are in equilibrium. This equilibrium can be displaced toward the pathologic form by genetic mutations, environmental fluctuations $(\mathrm{pH}$, presence of salt, metal ions, and free radicals etc.), changes in protein concentration, and/or chaperone proteins [16-18].

It has been widely accepted that there is a strong correlation between the protein aggregation and cytotoxicities [19-23]. Growing evidence has suggested that the generation of partially unfolded denatured forms represents a pivotal prerequisite for protein fibrillogenesis/aggregation [8,24-27]. It should be noted that, despite extensive investigations on amyloid fibril formation, the detailed molecular mechanism has remained far from complete.

Hen egg-white lysozyme, an enzyme with 129 amino acids that lyses the cell walls of bacteria, has been extensively studied. Structurally, the monomeric form of the lysozyme has four disulfide bonds and adopts mainly helical conformation ( $\sim 30 \% \alpha$-helix; $\sim 6 \% \beta$ sheet) [28]. It has been shown that hen egg-white lysozyme possesses $\alpha$-domain, with sequences of 1-39 and 89-129, and $\beta$-domains, with sequence of 40-88. Evidence indicated that, while only $-40 \%$ of the enzyme is identical in sequence to the human lysozyme, the form found to form amyloid fibrils and be responsible for hereditary non-neuropathic systemic amyloidosis [29], these two forms of lysozyme, nonetheless, retain a high degree of structural homology.

Urea has been widely used for protein folding or refolding studies [30,31]. In the current work, employing hen egg-white lysozymes as a model system, attempts were directed toward exploring the effects of protein partial unfolding on the formation of amyloid fibrillike species by denaturation in solutions with various urea concentrations at three different temperatures. Via fluorescence spectroscopies with ThT and ANS dyes, circular dichroism absorption spec- 
troscopy, Congo red binding assay, and electron microscopy, data reported here indicated that the amyloid fibril-like lysozyme species were first produced and then disappeared in solutions ( $\mathrm{pH} 7.4$ ) with $8 \mathrm{M}$ urea at all temperatures and with $4 \mathrm{M}$ urea at 45 or $55^{\circ} \mathrm{C}$; however, no fibrillar species were perceived in other conditions. In addition, surprisingly, even at the times with no or low-magnitude ThT fluorescence signal, SDS-PAGE data further suggested that certain larger unstructured aggregated species were formed at the later stage of the urea-induced unfolding process due to an unknown conformation switch. Our observations revealed that urea caused lysozymes to destabilize and temporarily form fibrils. We, therefore, conclude that there exists a close-knit relationship between the ureainduced lysozyme conformation and its propensity for fibrillization. We believe the results reported in this work, along with other studies, will advance our understanding toward the molecular mechanisms of protein denaturation and amyloid fibrillogenesis.

\section{MATERIALS AND METHODS}

\section{Proteins and Reagents}

Hen egg-white lysozyme (HEWL; EC 3.2.1.17) was purchased from Merck (Germany) and without further purification. Hydrochloric acid $(\mathrm{HCl})$ and di-potassium hydrogen phosphate $\left(\mathrm{K}_{2} \mathrm{HPO}_{4}\right)$ were obtained from Merck (Germany). Urea and potassium di-hydrogen phosphate $\left(\mathrm{KH}_{2} \mathrm{PO}_{4}\right)$ were obtained from Hayashi Pure Chemical Ind., Co., Ltd (Japan). All other chemicals, unless otherwise specified, were obtained from Sigma Chemical Co. (St. Louis, MO, USA).

\section{Lysozyme Sample Solution Preparation}

Sample solutions of $2 \mathrm{mg} / \mathrm{mL}$ HEWL were prepared by dissolving $0.1 \mathrm{~g}$ lyophilized HEWL in $50 \mathrm{~mL}$ of phosphate buffered saline (PBS, $136.7 \mathrm{mM} \mathrm{NaCl}, 2.68 \mathrm{mM} \mathrm{KCl}, 10 \mathrm{mM} \mathrm{Na}_{2} \mathrm{HPO}_{4}$, $1.76 \mathrm{mM} \mathrm{KH}_{2} \mathrm{PO}_{4}, \mathrm{pH} 7.2$ ) with $0.01 \%$ (w/v) sodium azide. HEWL sample solutions were first mixed via vortexing and then incubated at 37,45 , or $55^{\circ} \mathrm{C}$ during the course of experiment.

\section{BCA Protein Assay}

The lysozyme concentrations were verified with the bicinchoninic acid (BCA) assay [32].

\section{Lysozyme Enzyme Activity Assay}

The activity of lysozyme sample solutions was determined by measuring the decrease in absorbance at $450 \mathrm{~nm}$. Under the $\mathrm{pH}$ of 6.2 and $25^{\circ} \mathrm{C}$, a $2.5 \mathrm{~mL}$ volume of $M$. lysodeikticus suspension $(0.2$ $\mathrm{mg} / \mathrm{ml}$ ) in $0.06 \mathrm{M}$ potassium phosphate was used as the substrate solution to react with $40 \mu \mathrm{L}$ of protein sample solution. One unit of activity corresponds to an absorbance decrease of 0.001 per minute. The absorbance measurement was performed by using a Spectronic Genesys 5 spectrophotometer (Spectronic Instrument, USA).

\section{Thioflavin T Fluorescence (ThT) Assay}

$40 \mu \mathrm{L}$ of lysozyme samples taken at different times was mixed with $960 \mu \mathrm{L}$ of $10 \mathrm{mM}$ thioflavin $\mathrm{T}$ (ThT) in phosphate buffered saline (PBS) with $0.01(\mathrm{w} / \mathrm{v}) \%$ sodium azide. Samples were first loaded in a $1 \mathrm{~cm} \times 1 \mathrm{~cm} \times 4.5 \mathrm{~cm}$ polystyrene cuvette and ThT fluorescence intensity measurements were performed by exciting samples at $440 \mathrm{~nm}$ and recording emission intensities at $485 \mathrm{~nm}$ using an F-2500 Fluorescence Spectrophotometer (Hitachi, Japan). Slits were adjusted to $10 \mathrm{~nm}$ for both excitation and emission. The photomultiplier tube voltage was set to $400 \mathrm{~V}$. All measurements were taken in triplicate.

\section{Congo Red Binding Assay}

To assess the presence of amyloid fibrils in the lysozyme solutions, Congo red binding studies were performed. Congo red dye was dissolved in PBS to a final concentration of $112 \mu \mathrm{M}$. Congo red absorbances of lysozyme sample solutions and the free dye controls were determined by adding Congo red to a final concentration of $18 \mu \mathrm{M}$ and acquiring spectral measurements from 400 to 700 $\mathrm{nm}$ at $25^{\circ} \mathrm{C}$ by using a Spectronic Genesys 5 Spectrophotometer (Spectronic Instrument, USA) [33]. Both the lysozyme solutions and the control solutions were allowed to interact with Congo red for at least 30 minutes prior to recording of their spectra. All measurements were taken in triplicate.

\section{ANS-Binding Assay}

$50 \mu \mathrm{L}$ of lysozyme samples $(2 \mathrm{mg} / \mathrm{mL})$ in phosphate buffer salined solution was mixed with $900 \mu \mathrm{L}$ of $20 \mu \mathrm{M}$ 1-anilinonaphthalene-8-sulfonic acid (ANS) in PBS. Mixtures taken at different times were analyzed with an F-2500 Fluorescence Spectrophotometer (Hitachi, Japan). The excitation was set at $380 \mathrm{~nm}$ and the total ANS fluorescence emission was recorded between 420 and $580 \mathrm{~nm}$. The representative ANS fluorescence intensity was taken at the average emission wavelength (AEW), which accounts for both changes in intensity and spectrum envelope. The determination of AEW was carried out with the following equation:

$$
\mathrm{AEW}=\left(\Sigma \mathrm{F}_{i} \lambda_{i}\right) /\left(\Sigma \lambda_{i}\right)
$$

Where $F_{i}$ is the ANS fluorescence intensity at wavelength $\lambda_{i}$. All measurements were taken in triplicate.

\section{Circular Dichroism Absorption Spectroscopy}

Circular dichroism (CD) spectra of lysozyme samples were recorded on a JASCO J-715 (150-S Type) spectrometer (Sunway Scientific Corporation) at $25^{\circ} \mathrm{C}$ by using a bandwidth of $2.0 \mathrm{~nm}$, a step interval of $0.1 \mathrm{~nm}$, and an averaging time of 2 seconds. A $0.01 \mathrm{~cm}$ quartz cell was used for far-UV (190-260 nm) measurements. Three scans each of duplicate samples were measured and averaged. Control buffer scans were run in duplicate, averaged, and then subtracted from the sample spectra. The results were depicted in terms of ellipticity (millidegree) versus wavelength (nm).

\section{Transmission Electron Microscopy}

Samples were placed on carbon stabilized, formvar coated grids. Grids were negatively stained with $2 \%(\mathrm{w} / \mathrm{v})$ aqueous uranyl acetate (Electron Microscopy Sciences, Hatfield, PA, USA) and then examined and photographed in a JEOL, JEM-1200EX II transmission electron microscope (Tokyo, Japan) at an accelerating voltage of $80 \mathrm{kV}$.

\section{SDS-PAGE Electrophoresis}

SDS polyacrylamide gel electrophoresis (SDS-PAGE) was performed under the non-reducing condition (without mercaptoethnol). 5 volume of HEWL samples was mixed with 1 volume of $6 \times$ stock sample buffer ( $0.92 \mathrm{~g}$ SDS, $0.3 \mathrm{~g}$ Tris, $4 \mathrm{~g}$ glycerol, $2 \mathrm{ml} 2-$ mercaptoethanol, and $2 \mathrm{ml}$ bromophenol $\left(0.1 \%\right.$ in $\left.\left.\mathrm{H}_{2} \mathrm{O}\right), \mathrm{pH} 6.8\right)$. The resulting sample solutions were then applied to $15 \%$ Tris gels, and their electrophoretic separation was conducted in a Mini Protean II Electrophoresis system (Biorad, USA). The mixture of reference polypeptide markers was purchased from BioRad. Coomassie brilliant blue B7920 (Sigma, USA) (0.25 g Coomassie Brilliant Blue $\mathrm{R}-250$ in $125 \mathrm{~mL}$ methanol$/ 25 \mathrm{~mL}$ acetic acid/ $150 \mathrm{~mL}$ water, fil- 
tered before use) was used for staining and the following destaining step was performed by using destaining agent $\left(100 \mathrm{ml} \mathrm{CH}_{3} \mathrm{OH} /\right.$ $100 \mathrm{ml} \mathrm{CH} \mathrm{CHOH}_{3} \mathrm{CO0} \mathrm{ml}$ water) for around 2 hours with gentle agitation.

\section{RESULTS}

\section{Effect of Urea on HEWL Activity}

The effect of urea on hen egg-white lysozyme (HEWL) activity was investigated at three different temperatures: 55,45 , and $37^{\circ} \mathrm{C}$. With M. lysodeikticus suspension $(0.2 \mathrm{mg} / \mathrm{mL})$ as the substrate solution, the activity of lysozyme sample solutions was determined by measuring the decrease in absorbance at $450 \mathrm{~nm}$ with time ( 1 unit is equivalent to 0.001 absorbance decrease of 0.001 per minute). As depicted in Figs. 1A, 1B, and 1C, regardless of the incubation temperature in use, the activities of HEWL in phosphate buffer salined solutions (PBS) (pH 7.4) in the presence of urea were reduced markedly with incubation period. The higher the urea concentration added, the faster the level of activity dropped. On the contrary, for the native HEWL dissolved in PBS ( $\mathrm{pH}$ 7.4) without urea at 55,45 , or $37^{\circ} \mathrm{C}$, no obvious change in enzyme activity was observed even at 16 days after the initiation of incubation (see Figs. 1A, 1B, and $1 \mathrm{C}$ ). Our activity measurements suggested that the addition of urea into native HEWL solution led to a loss of enzyme activity in a urea concentration-dependent fashion.

\section{Effect of Urea on HEWL Structure}

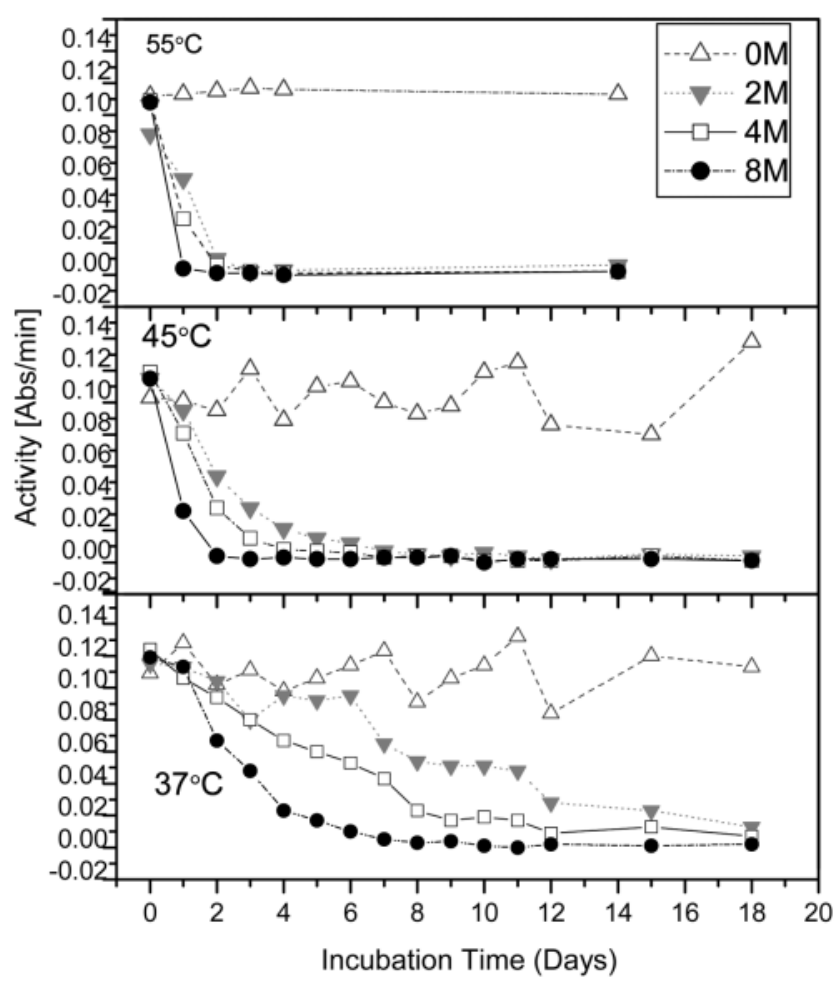

Fig. 1. The effect of urea on activity of hen egg-white lysozymes. The time-course of enzyme activity of hen egg-white lysozyme in the presence of $0,2,4$, or $8 \mathrm{M}$ was measured at (A) $55^{\circ} \mathrm{C}$; (B) $45^{\circ} \mathrm{C}$; or (C) $37^{\circ} \mathrm{C}$. Data represent the mean value of activity measurements of at least 5 independent experiments $(\mathrm{n} \geq \mathbf{5})$.
In studying the effect of urea on the structural change of HEWL, we monitored the ANS fluorescence emitted at the average emission wavelength upon excitation at $380 \mathrm{~nm}$ with incubation period. The ANS fluorescent dye has been frequently used to probe for conformational changes, hydrophobicity in particular, in proteins [34 36]. It has been shown that, upon preferential binding to the hydrophobic surfaces, the fluorescence intensity induced by ANS is enhanced accompanying a blue shift in the wavelength of the emission maximum owing to the extent of non-polar nature of the microenvironment of protein. As demonstrated in Fig. 2A, relative to the native HEWL alone, the addition of urea ( 4 or $8 \mathrm{M}$ ) to HEWL samples at $55^{\circ} \mathrm{C}$ led to a dramatic increase in the ANS fluorescence along with an abrupt blue shift in the emission wavelength for the first 1-2 days and then a decrease in signal was observed after the maximum intensity was reached ( $\sim 350$ units). Similarly, the presence of 4 or $8 \mathrm{M}$ urea resulted in an enhancement of ANS fluorescence emitted at $45^{\circ} \mathrm{C}$ (Fig. 2B). It was discovered that the extent of initial increase in fluorescence intensity was positively correlated with the urea concentration, i.e., $8 \mathrm{M}$ urea induced a relatively larger fluorescence enhancement than that by 2 or $4 \mathrm{M}$ urea at all temperatures in use. Furthermore, it was obvious that the time required for reaching the peak intensity decreased with increasing temperature of incubation at constant urea concentration (see Figs. 2A, 2B,

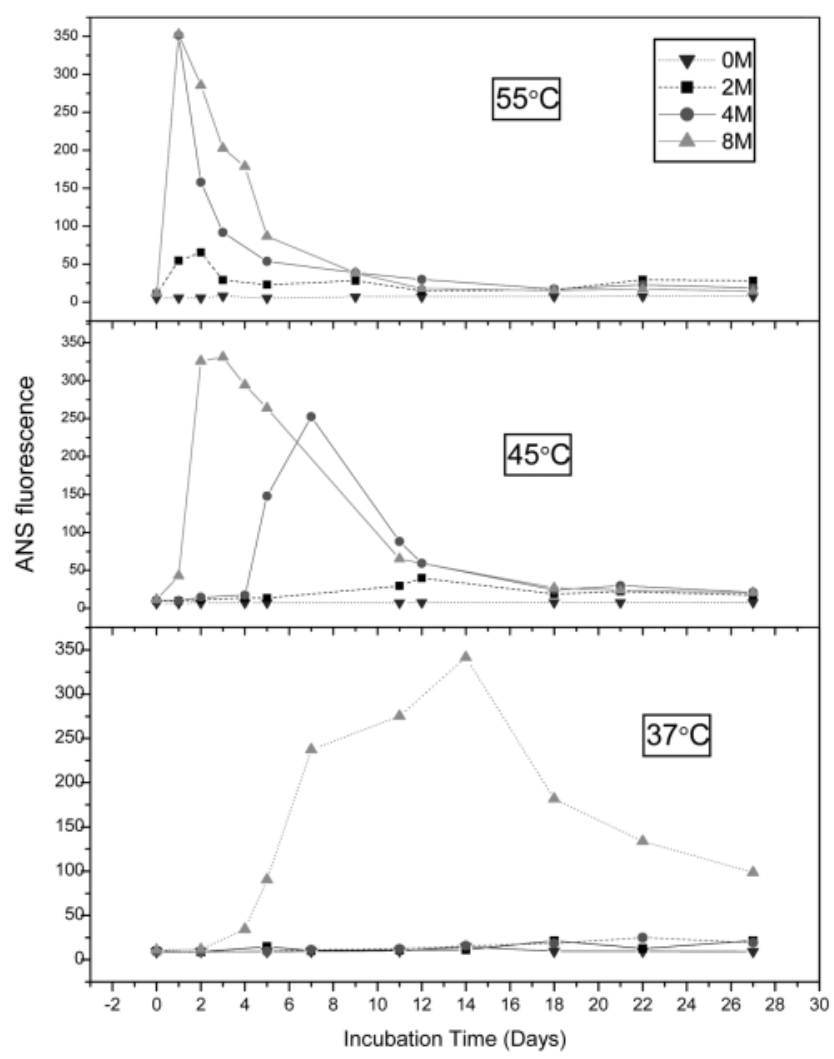

Fig. 2. The effect of urea on hydrophobicity of hen egg-white lysozymes during the course of aggregation. The time-course of hydrophobic property of hen egg-white lysozyme was measured by ANS-binding fluorescence at different temperatures $\left(55^{\circ} \mathrm{C}, 45^{\circ} \mathrm{C}\right.$ or $\left.37^{\circ} \mathrm{C}\right)$ in the presence of $0,2,4$, or $8 \mathrm{M}$ urea. Data were presented as an ANS fluorescence intensity taken at the average emission wavelength.

Korean J. Chem. Eng.(Vol. 24, No. 5) 
and $2 \mathrm{C}$ ). For example, with the addition of urea at $8 \mathrm{M}$, approximately 1,3 , and 14 days were needed to achieve the peak intensities for incubation temperatures at 55,45 , and $37^{\circ} \mathrm{C}$, respectively. Our data also indicated that there was almost no difference in ANS fluorescence emission between HEWLs without urea and with low urea concentration (e.g. $2 \mathrm{M}$ ).

\section{Effects of Urea on HEWL Amyloid Fibril Formation}

Two spectroscopic assays, ThT fluorescence spectroscopy and Congo red absorption spectroscopy, were utilized to examine the time-dependent formation of HEWL fibrils as a function of concentration of urea. ThT has been shown to interact specifically and rapidly with amyloid fibrils, and the primary structure of proteins has negligible effect on the binding between ThT and proteins [37]. An elevation in ThT fluorescence intensity has been reported as an important indicator of the presence of amyloid fibril [37]. As illustrated in Figs. 3A and 3B, for both $4 \mathrm{M}$ and $8 \mathrm{M}$ urea, the ThT fluorescence intensities at 55 and $45^{\circ} \mathrm{C}$ first climbed up and reached the maximum fluorescence signals within $\sim 2$ and $\sim 5$ days, respectively, and then dropped with increasing period of incubation. These results implied an initial elevation followed by a decrease of HEWL fibril formation. In addition, either at 55 or $45^{\circ} \mathrm{C}$, the maximum fluorescence signal of HEWL with $8 \mathrm{M}$ urea was approximately twice that with $4 \mathrm{M}$ urea, while a larger difference was observed

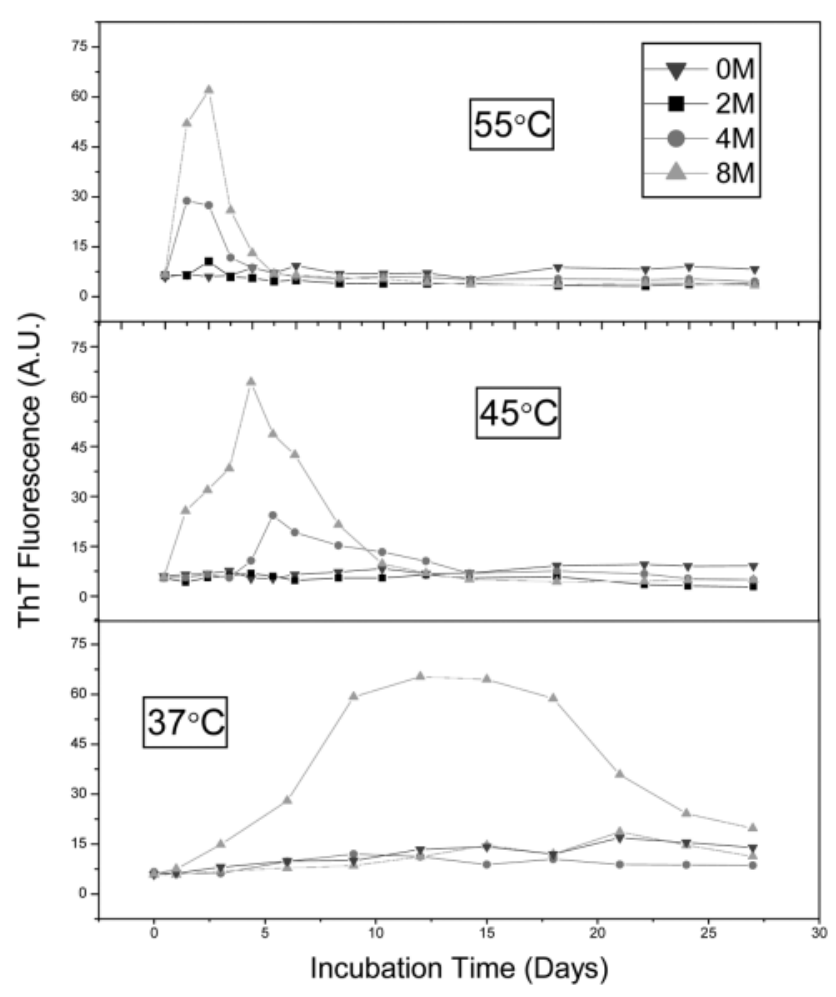

Fig. 3. The effect of urea on kinetics of fibril formation of hen eggwhite lysozymes. The extent of fibril formation was measured via ThT fluorescence as a function of incubation time of fibrillogenesis. Lysozymes were dissolved in phosphate buffered saline (pH 7.4) in the presence of 2,4 , and $8 \mathrm{M}$ at (A) $55^{\circ} \mathrm{C}$; (B) $45^{\circ} \mathrm{C}$; and (C) $37^{\circ} \mathrm{C}$. Samples at different incubation times were then tested for fibril formation. Data represent the mean ThT fluorescence measurement of at least 5 independent experiments $(n \geq 5)$. for the case of $37^{\circ} \mathrm{C}$ (see Figs. 3A, 3B, and 3C). However, in 0 or $2 \mathrm{M}$ urea, no significant ThT fluorescence emitted from HEWL solutions was observed during 27 days of incubation regardless of the temperature used, indicating that amyloid fibrils were not produced in the process (see Figs. 3A, 3B, and 3C). Our ThT fluorescence analysis was found to have a similar trend as the ANS fluorescence emission measurement shown previously. It is worth noting that, due to its short-lived nature, the species associated with the elevation of ThT fluorescence emission may be adequately named "amyloid fibril-like species".

Congo red absorbance spectroscopy was then utilized to probe for the presence of cross $\beta$-pleated sheet structure associated with amyloid fibrils. Irrespective of the incubation temperature used, HEWLs in PBS with $8 \mathrm{M}$ urea shifted the spectral properties of Congo red and exhibited a second shoulder peak at around $540 \mathrm{~nm}$, which is indicative of strong binding between HEWL and Congo red dye and generation of appreciable amount of amyloid fibrils. We quantitatively demonstrate the Congo red binding results by plotting the absorbance difference at a wavelength of $540 \mathrm{~nm}$ versus incubation period (Figs. 4A, 4B, and 4C). The HEWL solutions with $8 \mathrm{M}$ urea at all temperatures exhibited greater absorbance differences, implying higher degree of amyloid formation. Except for the case of $4 \mathrm{M}$ urea at $55^{\circ} \mathrm{C}$, HEWLs had essentially a zero absorbance

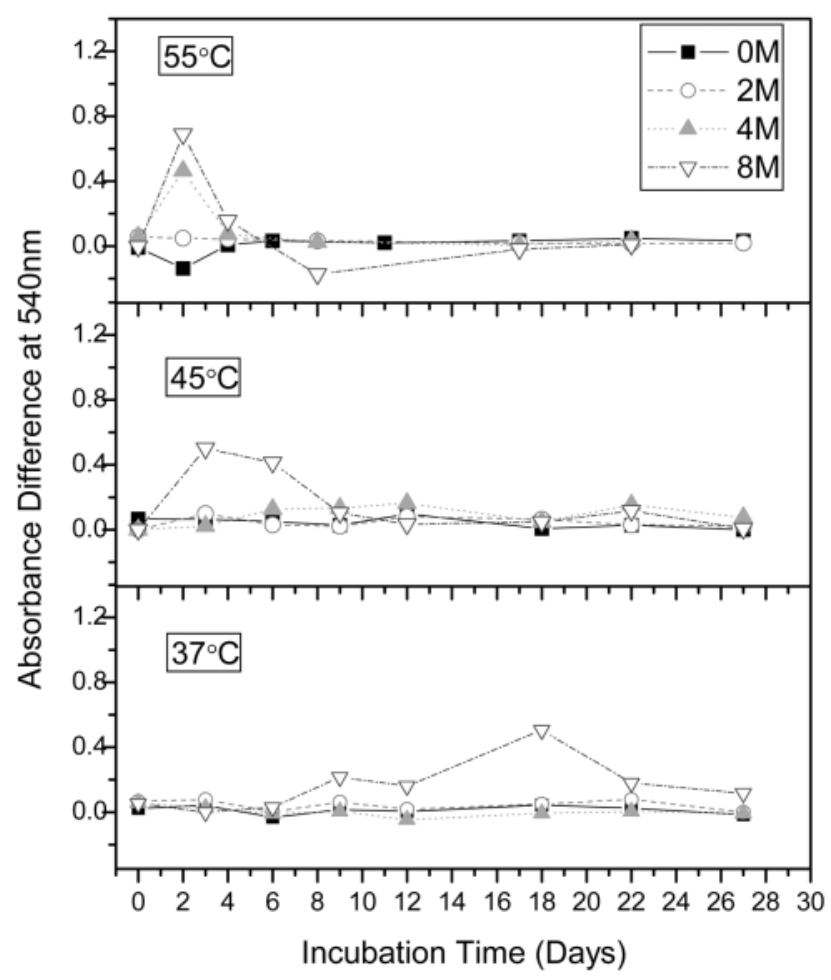

Fig. 4. The effect of urea on kinetics of amyloid fibril formation of hen egg-white lysozymes. Relative fibril formation was estimated via Congo red absorbance difference at $540 \mathrm{~nm}$ as a function of elapsed time of fibrillogenesis. Lysozymes were dissolved in phosphate buffered saline (pH 7.4) in the presence of 2, 4, and $8 \mathrm{M}$ at (A) $55^{\circ} \mathrm{C}$; (B) $45^{\circ} \mathrm{C}$; and (C) $37^{\circ} \mathrm{C}$. Measurements of Congo red absorbance difference at different incubation times were monitored for fibril formation. Data represent the mean value of measurements of at least 6 independent experiments $(n \geq 6)$. 
difference in other conditions $\left(0-4 \mathrm{M}\right.$ at 37 or $45^{\circ} \mathrm{C}, 0-2 \mathrm{M}$ at $\left.55^{\circ} \mathrm{C}\right)$, indicating that the protein did not bind Congo red and that negligible amount of amyloid fibrillar species associated with cross $\beta$-pleated sheet was detected under these conditions. Overall, the data demonstrated in Figs. 4A, 4B, and 4C are in accordance with the ones from our ThT fluorescence measurements (Figs. 3A, 3B, and 3C).

4. Morphological Evidence on Urea-Induced HEWL Aggregation and Amyloid Fibril Formation

The physical appearance of the HEWL solutions under various conditions was monitored during the course of experiment. A noticeable amount of aggregated species was observed at the bottom of the tubes for the cases of HEWLs with 2 and $4 \mathrm{M}$ urea at $45^{\circ} \mathrm{C}$ and $55^{\circ} \mathrm{C}$. At $45^{\circ} \mathrm{C}$ the urea-induced HEWL precipitation arose at 11 and 12 days after the onset of aggregation for $2 \mathrm{M}$ and $4 \mathrm{M}$ urea, respectively. Similarly, at $55{ }^{\circ} \mathrm{C}$ the precipitation occurred at 1 and 2 days for 2 and $4 \mathrm{M}$, respectively. However, when dissolved in PBS (pH 7.4) with urea at $8 \mathrm{M}$, the HEWL solution displayed visibly turbid solution without the formation of protein precipitates/pellets. In addition, no precipitates were found at all concentrations of urea when incubation temperature was set at $37^{\circ} \mathrm{C}$. We quantitatively
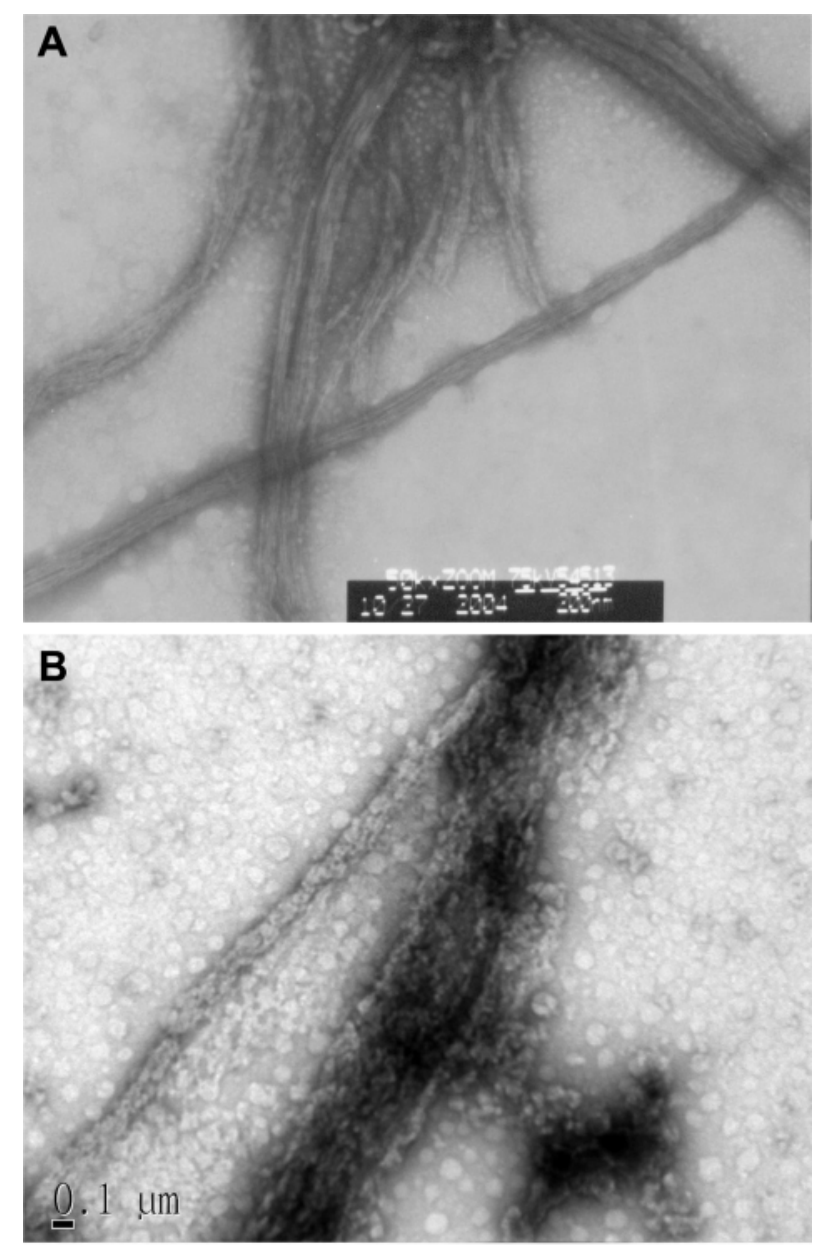

Fig. 5. Electron micrographs of negatively stained hen egg-white lysozymes. Lysozyme samples were prepared in phosphate buffered saline ( $\mathrm{pH} 7.4$ ) with $8 \mathrm{M}$ urea at $55^{\circ} \mathrm{C} 2$ days after the initiation of aggregation (A) and with $8 \mathrm{M}$ urea at $45^{\circ} \mathrm{C}$ 5 days after the initiation of aggregation (B). analyzed the ability of urea to induce precipitation of HEWLs by measuring the protein concentrations in the supernatants of samples under various conditions. Our results showed that almost no proteins were lost in HEWL samples at $37{ }^{\circ} \mathrm{C}$ regardless of urea concentration in use (data not shown). The maximal losses of protein, $\sim 73 \%$ after 5 days and $\sim 11 \%$ after 12 days, were recorded when HEWLs were incubated in $2 \mathrm{M}$ urea at $55^{\circ} \mathrm{C}$ and $2 \mathrm{M}$ urea at $45^{\circ} \mathrm{C}$, respectively (data not shown). This is further confirmation that the urea-induced fibrillogenesis came from the transmission electron micrographs of amyloid fibrils formed from HEWLs in the presence of $8 \mathrm{M}$ urea at $55^{\circ} \mathrm{C}$ and $45^{\circ} \mathrm{C}$ as illustrated in Figs. 5A and $5 \mathrm{~B}$, respectively (similar EM results were found in samples with $8 \mathrm{M}$ urea incubated at $37^{\circ} \mathrm{C}$ ). The morphology of aggregated species observed in these preparations was reminiscent of typical amyloid fibrils with $\sim 10 \mathrm{~nm}$ in diameter and $\sim \mu \mathrm{m}$ in length.

To explore the size distribution of species formed in the solution during the fibrillization process, we performed SDS-PAGE electrophoresis on HEWL sample solutions at different urea concentrations. When subjected to 4 or $8 \mathrm{M}$ urea at $55^{\circ} \mathrm{C}$ for 2 days (Fig. 6A), $8 \mathrm{M}$ at $45^{\circ} \mathrm{C}$ for 5 days (Fig. 6B), and $8 \mathrm{M}$ at $37^{\circ} \mathrm{C}$ for 14 days (figure not shown), there apparently existed HEWL species migrating with molecular weights greater than monomeric mass $(14.3 \mathrm{kDa})$ (see Lanes 4 and 5 in Fig. 6A; Lane 5 in Fig. 6B). In contrast, HEWL in other conditions showed no evidence of macromolecular species or species that migrated with molecular weights greater than $14.3 \mathrm{kDa}$. These PAGE results verified and supported the presence of larger fibrillar/aggregated species suggested by our results from spectroscopic analyses stated earlier. SDS-PAGE electrophoresis was also carried out at the later stage (the time points with low-magnitude ThT signal) of the aggregation process in all experimental conditions. As can be seen in Figs. 6C and 6D, evidently, even with almost zero ThT fluorescence intensities, some larger species of HEWL were present in $8 \mathrm{M}$ urea solution at $45^{\circ} \mathrm{C}$ after 16 days and in $4 \mathrm{M}$ or $8 \mathrm{M}$ urea solution at $37^{\circ} \mathrm{C}$ after 27 days of incubation. These results suggested that, while the cause has yet been clarified, certain conditions prompted the temporary formation of HEWL amyloid fibrils that ultimately converted into unstructured aggregates.

\section{Effect of Urea on HEWL Conformation}

To elucidate the role of urea in the structural changes of HEWL, CD absorption spectroscopy was employed to investigate the secondary structural transition. As pointed out by other reports, a detailed examination of the structure of HEWL samples via CD would not be possible owing to the presence of insoluble particulate matter in our samples [38]. As a result, qualitative structural information on the nature of HEWL samples was achieved by examining their suspensions or supernatants. As Fig. 7A attests, even at day 28 after the initiation of aggregation, the CD spectra of HEWL alone in PBS ( $\mathrm{pH}$ 7.4) at either incubation temperature showed a secondary structure with a shoulder at $\sim 222 \mathrm{~nm}$ as well as an absorption minimum at $\sim 208 \mathrm{~nm}$, reflecting an $\alpha$-helix-rich conformation. Similar trend in CD spectra was observed for HEWL plus $2 \mathrm{M}$ or $4 \mathrm{M}$ urea at $37^{\circ} \mathrm{C}$, HEWL plus $2 \mathrm{M}$ urea at $45^{\circ} \mathrm{C}$, and HEWL plus $2 \mathrm{M}$ urea at $55^{\circ} \mathrm{C}$. On the contrary, HEWLs with $8 \mathrm{M}$ at all temperatures or with $4 \mathrm{M}$ urea at $55^{\circ} \mathrm{C}$ and $45^{\circ} \mathrm{C}$ permitted an occurrence of structural transition, resulting in a striking alteration in the relative proportion of secondary structures (see Figs. 7B-7F). Obviously, the 
A

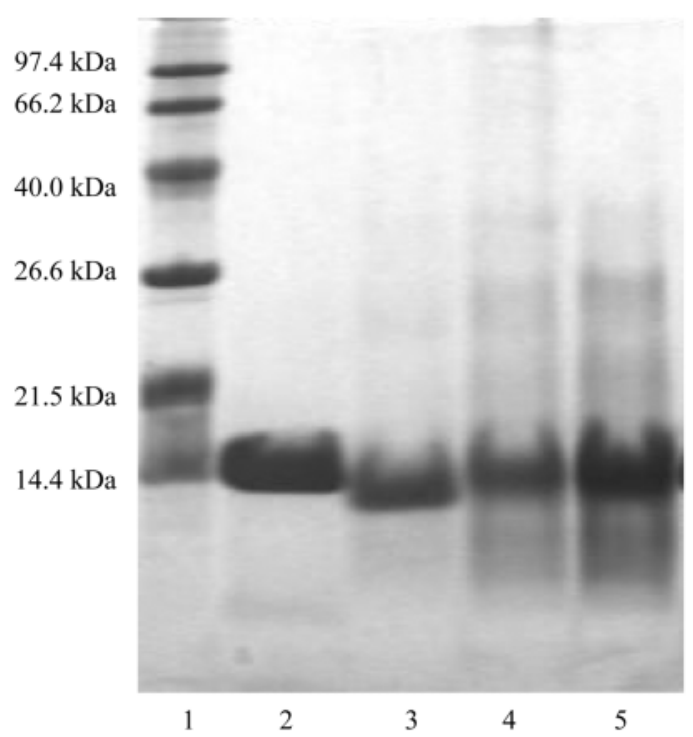

C

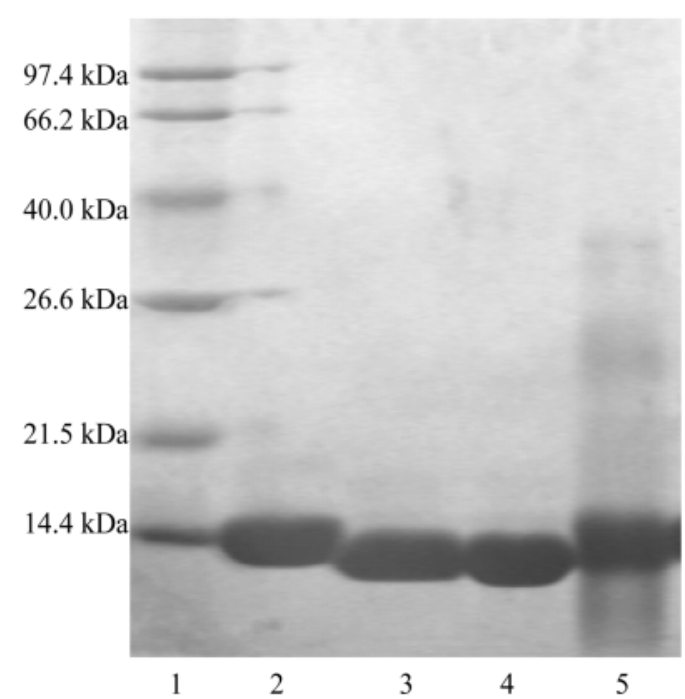

B

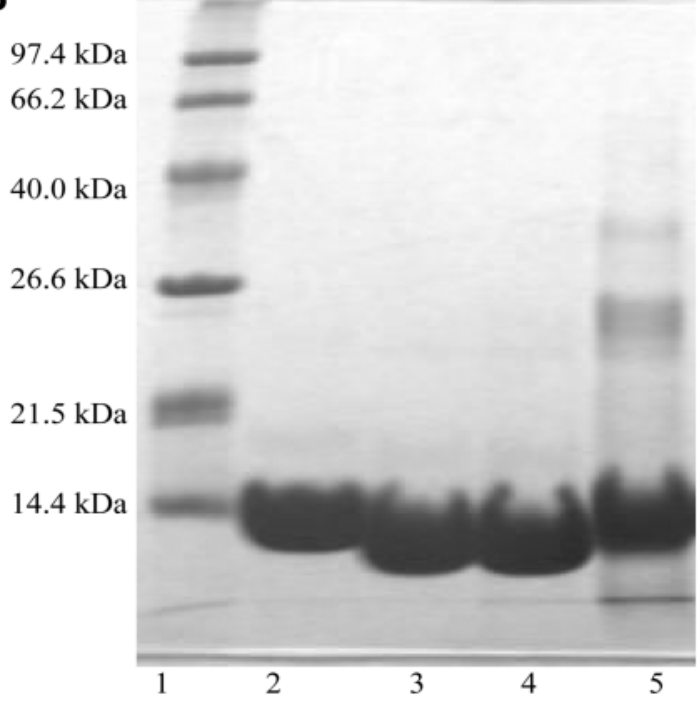

D

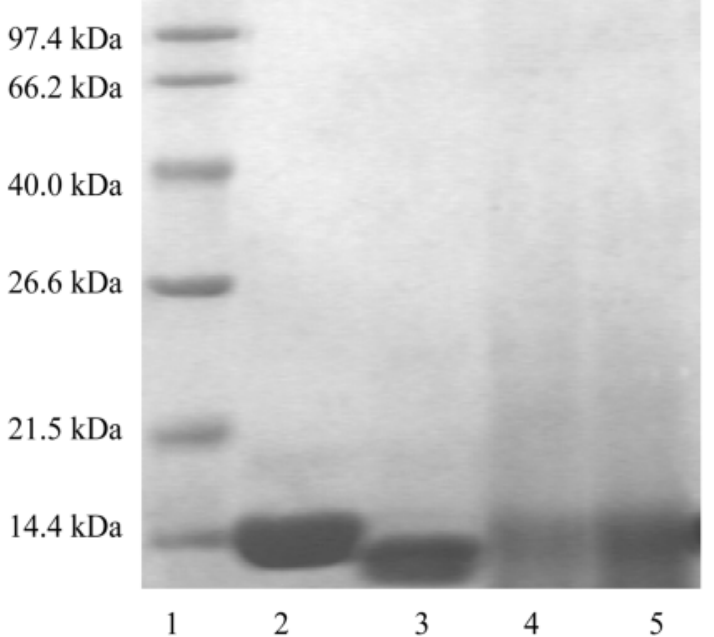

Fig. 6. Representative SDS polyacrylamide gel electrophoresis of hen egg-white lysozymes. (A) Lysozyme samples were incubated at $55{ }^{\circ} \mathrm{C}$ for 2 days: Lane 1: molecular weight markers; Lane 2: with 0 M urea; Lane 3: with 2 M urea; Lane 4: with 4 M urea; Lane 5: with $8 \mathrm{M}$ urea. (B) Lysozyme samples were incubated at $45^{\circ} \mathrm{C}$ for 5 days: Lane 1: molecular weight marker; Lane 2: without urea; Lane 3: with $2 \mathrm{M}$ urea; Lane 4: with $4 \mathrm{M}$ urea; Lane 5: with $8 \mathrm{M}$ urea. (C) Lysozyme samples were incubated at $45^{\circ} \mathrm{C}$ for 16 days: Lane 1: molecular weight marker; Lane 2: without urea; Lane 3: with $2 \mathrm{M}$ urea; Lane 4: with $4 \mathrm{M}$ urea; Lane 5: with $8 \mathrm{M}$ urea. (D) Lysozyme samples were incubated at $37^{\circ} \mathrm{C}$ for 26 days: Lane 1: molecular weight marker; Lane 2: without urea; Lane 3: with 2 M urea; Lane 4: with 4 M urea; Lane 5: with $8 \mathrm{M}$ urea.

rate of structural transition appeared to positively correlate with the temperature or urea concentration. For example, after $\sim 2, \sim 5$, or $\sim 11$ days of incubation with $8 \mathrm{M}$ urea at $55^{\circ} \mathrm{C}, 45^{\circ} \mathrm{C}$, or $37^{\circ} \mathrm{C}$, respectively, the sample no longer displayed a helical structure. Instead, the sample exhibited the characteristic pattern of $\beta$-sheet-rich conformation with an absorption minimum around $\sim 220-222 \mathrm{~nm}$ (see Figs. 7C, 7E, and 7F). The timing for this $\alpha$-to- $\beta$ transition seemed to be consistent with that of the amyloid formation demonstrated in both ThT fluorescence and Congo red assays. Interestingly, as evident by the CD spectra, subsequent structural changes obtained in the conditions of higher urea concentrations were further detected as incubation time increased, implying the occurrence of a certain conversion switching away from a typical $\beta$-sheet-rich conforma- tion to a less ordered structure.

\section{DISCUSSION}

As of now, more than twenty different disease-related proteins including $\beta$-amyloid, human lysozyme, amylin, and $\alpha$-synuclein have been reported to misfold to form amyloid fibrils leading to their accompanying pathologies $[5,7,8,17,39,40]$. These proteins share little similarities in amino acid composition and sequence, yet they are able to polymerize into fibrillar species with a characteristic cross $\beta$-sheet secondary structure. Moreover, mounting evidence has suggested that non-disease associated proteins, under appropriate conditions, can aggregate in vitro to form amyloid fibril- 

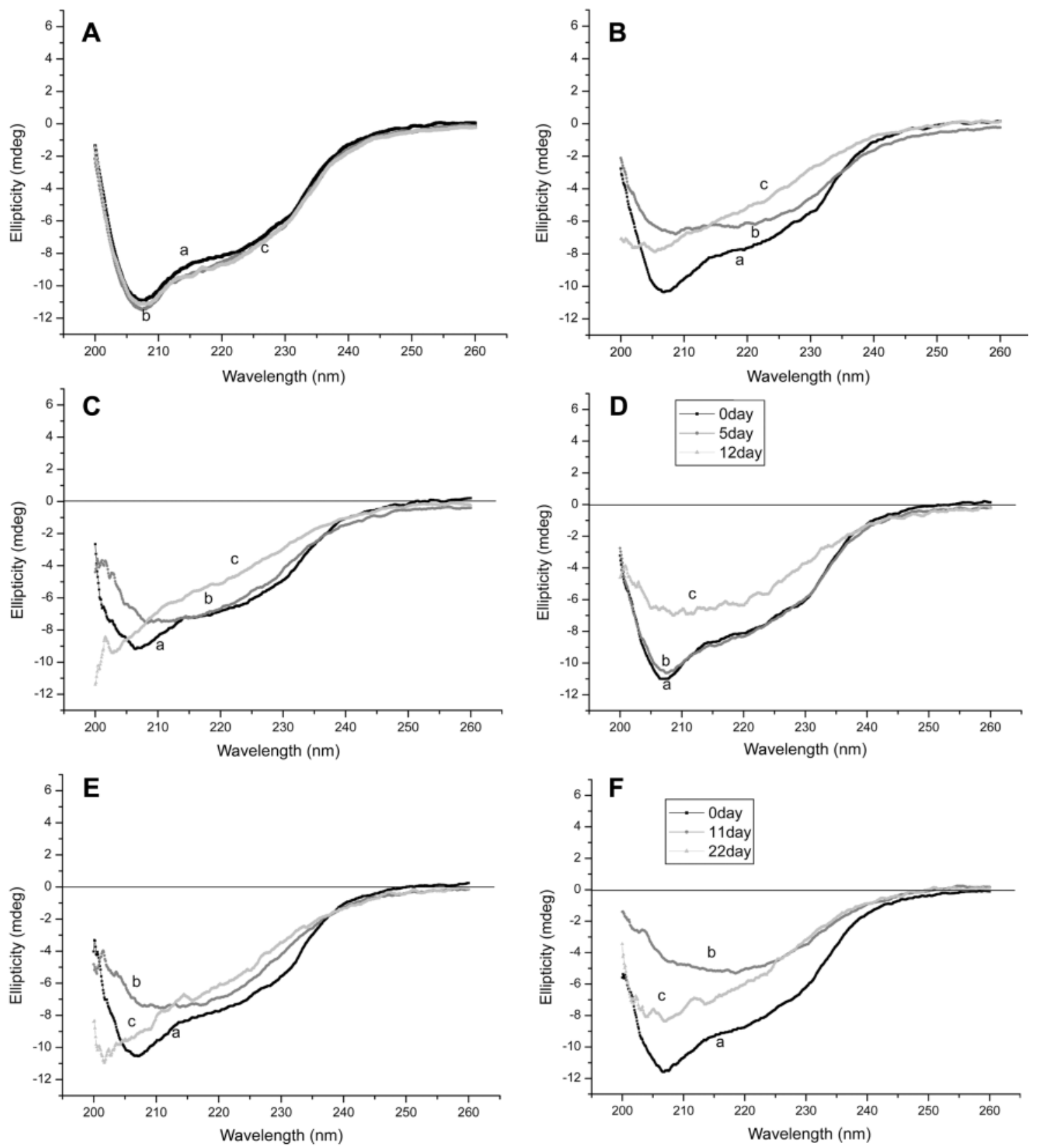

Fig. 7. (A) Representative CD spectra of hen egg-white lysozymes dissolved in phosphate buffered saline (pH 7.4 ) at $55{ }^{\circ} \mathrm{C}$ without urea taken at different incubation times (a: 0 day, b: 14 days, and c: 21 days). (B) Representative CD spectra of hen egg-white lysozymes dissolved in phosphate buffered saline (pH 7.4) at $55^{\circ} \mathrm{C}$ with $4 \mathrm{M}$ urea taken at different incubation times (a: 0 day, b: 2 days, and c: 5 days). (C) Representative $\mathrm{CD}$ spectra of hen egg-white lysozymes dissolved in phosphate buffered saline (pH 7.4$)$ at $55{ }^{\circ} \mathrm{C}$ with $8 \mathrm{M}$ urea taken at different incubation times (a: 0 day, b: 2 days, and c: 5 days). (D) Representative CD spectra of hen eggwhite lysozymes dissolved in phosphate buffered saline (pH 7.4) at $45^{\circ} \mathrm{C}$ with $4 \mathrm{M}$ urea taken at different incubation times (a: 0 day, b: 5 days, and c: 12 days). (E) Representative CD spectra of hen egg-white lysozymes dissolved in phosphate buffered saline (pH 7.4) at $45^{\circ} \mathrm{C}$ with $8 \mathrm{M}$ urea taken at different incubation times (a: 0 day, b: 5 days, and c: 12 days). (F) Representative CD spectra of hen egg-white lysozymes dissolved in phosphate buffered saline (pH 7.4) at $37^{\circ} \mathrm{C}$ with $8 \mathrm{M}$ urea taken at different incubation times (a: 0 day, b: 11 days, and c: 22 days).

lar species [9-11,41]. There is a wealth of data demonstrating that aggregation or amyloid fibril formation occurs when the native protein structure is destabilized and experiences a conformational change leading to a partially unfolded intermediate species. The formation of partially unfolded species has been widely viewed as a structural prerequisite for fibril formation $[8,24-27,42]$.
Several lines of evidence suggest that the presence of denaturants has an impact on the formation of amyloid species and aggregates as well as their conformational changes. McDuff et al. reported that the formation of alpha-urease aggregates and fibril-like structures was observed under the electron microscope in the presence of a moderate concentration of guanidine hydrochloride (0.5-1.5 M), 
while urease hexamer particles, round in shape and with a diameter of $13 \mathrm{~nm}$, were found in the absence of guanidine hydrochloride [43]. Moreover, Nielsen et al. found that by adding increasing concentrations of urea to a fixed concentration of insulin at $\mathrm{pH} 7.4$ resulted in a decrease in lag time and, inversely, a slight increase in elongation rate constants. However, the opposite trend was observed in the presence of stabilizers, TMAO and sucrose, indicating the importance of the buildup of partially folded intermediates during the lag phase [44]. Aside from utilizing denaturants, other researchers also performed denaturation on proteins by creating mutations or varying $\mathrm{pH}$ conditions $[45,46]$. Their results demonstrated a strong link between the formation of partially folded conformers and the occurrence of protein fibrillization or aggregation [47-49]. Notably, the detailed molecular mechanism in regards to how partially unfolded intermediates undergo conformational transition from the native state to the amyloid fibrils remained far from complete.

Vernaglia and coworkers demonstrated that hen lysozymes were partially unfolded and rapidly formed fibrils when incubated with guanidine hydrochloride concentrations at 2-5 M [38]. Their results also indicated that the conditions with extremely low or extremely high concentrations of guanidine hydrochloride were not able to induce fibrils [38]. To date, nevertheless, little has been reported in regards to the production of HEWL amyloid fibril and its relevant mechanism(s) by urea-induced denaturation or unfolding.

In the present study, we have used urea to induce denaturation and subsequent progressive aggregation of HEWL. We further examined the effects of urea on the aggregation process at 37,45 , and $55^{\circ} \mathrm{C}$. Conditions in which HEWL enzyme activity was decreased by urea were first identified. Our data showed that the reduction in HEWL activity was highly dependent upon urea concentration. We then demonstrated that HEWLs formed amyloid fibrils under certain conditions, such as $8 \mathrm{M}$ urea at 37,45 , and $55^{\circ} \mathrm{C}$ as well as $4 \mathrm{M}$ urea at 45 and $55^{\circ} \mathrm{C}$ through ThT fluorescence enhancement (Fig. 3), Congo red binding assay (Fig. 4), and transmission electron microscopy (Fig. 5). Very low concentration of urea (e.g., $2 \mathrm{M}$ ), however, failed to induce the production of HEWL fibrils. Our results further implied that the fibrils either disappeared or transformed into other unordered conformers when the incubation time was prolonged. Via ANS fluorescence and CD spectroscopy, we found that, in the presence of $4 \mathrm{M}$ or $8 \mathrm{M}$ urea, the native lysozyme with essentially very little solvent-exposed hydrophobic surfaces and a predominantly helical structure was first converted to a conformer adopting a $\beta$-sheet rich aggregated amyloid form. Next, with increasing incubation period, the $\beta$-sheet poor and un-ordered HEWL species with fewer solvent-exposed hydrophobic regions were detected. As supported by non-denaturing SDS electrophoresis, our results showed evidence of macromolecular species that migrated with molecular weights greater than $14.3 \mathrm{kDa}$ under the conditions in which HEWL adopted a $\beta$-sheet rich, aggregated, amyloid form. Interestingly, further exposure of HEWL to urea ( $4 \mathrm{M}$ or $8 \mathrm{M})$ resulted in the formation of a range of species with higher molecular weights, suggesting the possibility of a structural transformation whereby the $\beta$-sheet rich, aggregated conformer underwent a conformational transition into $\beta$-sheet poor, non-aggregated species. These phenomena are significantly different from those found in the studies using guanidine hydrochloride for denaturing HEWLs [38].

In conclusion, we have explored the effects of partial unfolding of HEWL on amyloid fibril formation by denaturation in solutions with various urea concentrations at three different temperatures. The results presented here detail how urea affects the secondary and tertiary structures of the native HEWL and assists in the formation of amyloid fibril-like HEWLs. We have successfully shown the induction of amyloid fibrils by urea under certain conditions; however, the presence of amyloid fibrils was only temporary. Our data suggest that a conformational change, while its mechanism remains unclear, exists whereby the structured amyloid-form HEWLs shift toward the unstructured non-amyloid-form conformers. While further investigation on the aforementioned unidentified conformational switch remains to be elaborated in full, our results undoubtedly support the notion that partial denaturation/unfolding is crucial to fibril formation. Nevertheless, it is our belief that the outcome from this work will enable us to comprehend the mechanism(s) of amyloid protein aggregation process associated with denaturation.

\section{ACKNOWLEDGMENT}

This work was supported by grants from the National Science Council, Taiwan. The authors would like to thank Dr. Rita P.-Y. Chen from Institute of Biochemistry at Academia Sinica, Taiwan for the assistance of circular dichroism measurements.

\section{REFERENCES}

1. A. L. Fink, Physiol. Rev., 79, 425 (1999).

2. W. A. Houry, Curr. Protein. Pept. Sci., 2, 227 (2001).

3. K. A. Markossian and B. I. Kurganov, Biochemistry (Mosc), 69, 971 (2004).

4. J. C. Young, V. R. Agashe, K. Siegers and F. U. Hartl, Nat. Rev. Mol. Cell Biol., 5, 781 (2004).

5. C. M. Dobson, Semin. Cell Dev. Biol., 15, 3 (2004).

6. R. M. Murphy, Annu. Rev. Biomed. Eng., 4, 155 (2002).

7. C. A. Ross and M. A. Poirier, Nat. Med., 10 Suppl, S10 (2004).

8. V. N. Uversky and A. L. Fink, Biochim. Biophys. Acta, 1698, 131 (2004).

9. Z. Lai, W. Colon and J. W. Kelly, Biochemistry, 35, 6470 (1996).

10. A. D. Ferrao-Gonzales, S. O. Souto, J. L. Silva and D. Foguel, Proc. Natl. Acad. Sci. USA, 97, 6445 (2000).

11. V. Sluzky, A. M. Klibanov and R. Langer, Biotechnol. Bioeng., 40, 895 (1992).

12. G H. Beaven, W. B. Gratzer and H. G Davies, Eur. J. Biochem., 11, 37 (1969).

13. F. Chiti, P. Webster, N. Taddei, A. Clark, M. Stefani, G Ramponi and C. M. Dobson, Proc. Natl. Acad. Sci. USA, 96, 3590 (1999).

14. M. Fandrich, V. Forge, K. Buder, M. Kittler, C. M. Dobson and S. Diekmann, Proc. Natl. Acad. Sci. USA, 100, 15463 (2003).

15. Y. Kallberg, M. Gustafsson, B. Persson, J. Thyberg and J. Johansson, J. Biol. Chem., 276, 12945 (2001).

16. S. R. Ji, Y. Wu and S. F. Sui, Gen. Physiol. Biophys., 21, 415 (2002).

17. P. T. Lansbury, Jr., Proc. Natl. Acad. Sci. USA, 96, 3342 (1999).

18. C. Soto, J. Mol. Med., 77, 412 (1999).

19. M. Stefani and C. M. Dobson, J. Mol. Med., 81, 678 (2003).

20. S. S. S. Wang and T. A. Good, J. Chin. Inst. Chem. Eng., 36, 533 (2005).

21. F. G De Felice, M. N. Vieira, L. M. Saraiva, J. D. Figueroa-Villar, 
J. Garcia-Abreu, R. Liu, L. Chang, W. L. Klein and S. T. Ferreira, Faseb. J., 18, 1366 (2004).

22. D. Schuster, A. Rajendran, S. W. Hui, T. Nicotera, T. Srikrishnan and M. L. Kruzel, Neuropeptides, (2005).

23. S. S. Wang, T. A. Good and D. L. Rymer, Int. J. Biochem. Cell Biol., 37, 1656 (2005).

24. J. C. Rochet, K. A. Conway and P. T. Lansbury, Jr., Biochemistry, 39, 10619 (2000).

25. E. Zerovnik, European Journal of Biochemistry, 269, 3362 (2002).

26. C. M. Dobson, Philos. Trans. R. Soc. Lond. B. Biol. Sci., 356, 133 (2001).

27. S. Ohnishi and K. Takano, Cell Mol. Life Sci., 61, 511 (2004).

28. M. C. Vaney, S. Maignan, M. Ries-Kautt and A. Ducriux, Acta. Crystallogr. D. Biol. Crystallogr., 52, 505 (1996).

29. M. B. Pepys, P. N. Hawkins, D. R. Booth, D. M. Vigushin, G. A. Tennent, A. K. Soutar, N. Totty, O. Nguyen, C. C. Blake, C. J. Terry and et al., Nature, 362, 553 (1993).

30. B. E. Fischer, Biotechnol. Adv., 12, 89 (1994).

31. Y. G. Gao, Y. X. Guan, S. J. Yao and M. G. Cho, Korean J. Chem. Eng., 19, 871 (2002).

32. P. K. Smith, R. I. Krohn, G. T. Hermanson, A. K. Mallia, F. H. Gartner, M. D. Provenzano, E. K. Fujimoto, N. M. Goeke, B. J. Olson and D. C. Klenk, Anal. Biochem., 150, 76 (1985).

33. W. E. Klunk, J. W. Pettegrew and D. J. Abraham, J. Histochem. Cytochem., 37, 1273 (1989).

34. C. P. Liu,Z. Y. Li, G C. Huang, S. Perrett and J. M. Zhou, Biochimie., 87, 1023 (2005).
35. A. L. Smoot, M. Panda, B. T. Brazil, A. M. Buckle, A. R. Fersht and P. M. Horowitz, Biochemistry, 40, 4484 (2001).

36. I. Sirangelo, E. Bismuto, S. Tavassi and G Irace, Biochim. Biophys. Acta., 1385, 69 (1998).

37. H. LeVine, 3rd, Protein. Sci., 2, 404 (1993).

38. B. A. Vernaglia, J. Huang and E. D. Clark, Biomacromolecules, 5, 1362 (2004).

39. J. W. Kelly, Curr. Opin. Struct. Biol., 8, 101 (1998).

40. J. D. Sipe, Crit. Rev. Clin. Lab. Sci., 31, 325 (1994).

41. V. Sluzky, J. A. Tamada, A. M. Klibanov and R. Langer, Proc. Natl. Acad. Sci. USA, 88, 9377 (1991).

42. A. Quintas, D. C. Vaz, I. Cardoso, M. J. Saraiva and R. M. Brito, J. Biol. Chem., 276, 27207 (2001).

43. F. O. McDuff, A. Doucet and M. Beauregard, Biochem. Cell Biol., 82, 305 (2004).

44. L. Nielsen, R. Khurana, A. Coats, S. Frokjaer, J. Brange, S. Vyas, V. N. Uversky and A. L. Fink, Biochemistry, 40, 6036 (2001).

45. V. N. Uversky, J. Li and A. L. Fink, J. Biol. Chem., 276, 10737 (2001).

46. P. O. Souillac, V. N. Uversky, I. S. Millett, R. Khurana, S. Doniach and A. L. Fink, J. Biol. Chem., 277, 12657 (2002).

47. R. Khurana, J. R. Gillespie, A. Talapatra, L. J. Minert, C. IonescuZanetti, I. Millett and A. L. Fink, Biochemistry, 40, 3525 (2001).

48. J. Goers, S. E. Permyakov, E. A. Permyakov, V. N. Uversky and A. L. Fink, Biochemistry, 41, 12546 (2002).

49. M. A. Speed, T. Morshead, D. I. Wang and J. King, Protein. Sci., 6, 99 (1997). 